\title{
Heat-Mapping: A Robust Approach Toward Perceptually Consistent Mesh Segmentation
}

\author{
Yi Fang \\ Purdue University \\ West Lafayette, IN 47907 \\ fang 4 @purdue. edu
}

\author{
Mengtian Sun \\ Purdue University \\ West Lafayette, IN 47907 \\ sun84@purdue. edu \\ Karthik Ramani \\ Purdue University \\ West Lafayette, IN 47907 \\ ramaniepurdue.edu
}

\author{
Minhyong Kim \\ University of College London \\ Gower Street, London WCIE, UK \\ minhyong.kimeucl.ac.uk
}

\begin{abstract}
$3 D$ mesh segmentation is a fundamental low-level task with applications in areas as diverse as computer vision, computer-aided design, bio-informatics, and $3 D$ medical imaging. A perceptually consistent mesh segmentation (PCMS), as defined in this paper is one that satisfies 1) invariance to isometric transformation of the underlying surface, 2) robust to the perturbations of the surface, 3) robustness to numerical noise on the surface, and 4) close conformation to human perception. We exploit the intelligence of the heat as a global structure-aware message on a meshed surface and develop a robust PCMS scheme, called Heat-Mapping based on the heat kernel. There are three main steps in Heat-Mapping. First, the number of the segments is estimated based on the analysis of the behavior of the Laplacian spectrum. Second, the heat center, which is defined as the most representative vertex on each segment, is discovered by a proposed heat center hunting algorithm. Third, a heat center driven segmentation scheme reveals the PCMS with a high consistency towards human perception. Extensive experimental results on various types of models verify the performance of Heat-Mapping with respect to the consistent segmentation of articulated bodies, the topological changes, and various levels of numerical noise.
\end{abstract}

\section{Introduction}

\subsection{Background}

In recent years, we have seen an explosive growth of the available 3D model data across a variety of fields, such as reverse engineering and 3D medical imaging, with the development of the acquisition techniques [1, 23, 20, 16, 15, 8,
$7,5,10,18]$. We are therefore faced with an ever-increasing demand for approaches towards automatic model processing, understanding and analysis. As a first important step of mesh model processing, the segmentation of a model into a small number of meaningful components is difficult. A segmentation approach likely confronts the problems of 1) isometrically-variant segmentation of the surface, 2) sensitivity to topological perturbations of the surface, 3 ) sensitivity to numerical noise inherently embedded within observed models, and 4) inconsistency with human understanding of segmentation. We define a segmentation, which can robustly provide a solution to the above four challenges above, as the perceptually consistent mesh segmentation (PCMS). Over the past several years, the integrated characteristics of PCMS has become more important for advanced mesh processing and understanding as it provides more insights into mesh models [8, 16, 23, 25]. PCMS facilitates the interpretation of 3D surface meshes in terms of either a pure geometric sense, semantic information or both through the representation of an intrinsically hidden geometric structure of the meshes. The intrinsic interpretation of the structure is able to partition the objects into a number of functional components in a way close to human understanding. Compared to ongoing 3D segmentation, PCMS is much more adequate to analyze the features of models, enabling diverse applications to modeling. This includes 3D shape (or partial shape) matching, skeleton extraction, texture mapping, simplification, parameterization and shape retrieval $[7,13,8,24]$.

\subsection{Brief review of related works}

Some studies have addressed how the mesh surface can be decomposed into perceptually meaningful units in an au- 
tomatic or semi-automatic way [8, 16, 23, 25]. The outreaching goal of a robust PCMS is to perform it on a model in an assumption free manner. While the previous approaches have shown the efficiency of addressing PCMS to some extent, there are some common limitations that have not been addressed for this challenging problem [8, 25, 9]. Here, we focus on the limitations arising from the dependence of prior generic assumptions about the shape of the $3 \mathrm{D}$ object like the number of segments or choice of initial seed points for segmentation or both. Mesh segmentation is a classical problem in low-level vision tasks for multimedia data processing. There is a great body of literature proposed for segmenting meshes $[7,2,8,16,23,25]$. As the focus of this paper is characterization of PCMS, our scope of review is limited to closely related works. The readers are referred to $[2,7,24,19,27,21]$ about general mesh segmentation approaches. The importance of the PCMS has been highlighted in some recent works $[8,16,23,25,11]$.

We roughly break the related works into two categories: non-diffusion and diffusion-based segmentation. The nondiffusion category [15] deals with segmentation using feature points and core extraction. The authors describe a pose oblivious mesh segmentation with a hierarchical representation of the structure of an object. However, because the feature points are sensitive to topological short-circuits and high numerical noise, a satisfying segmentation can not be easily obtained. The methods described in the later group are more recent and are oriented towards PCMS. These methods exploit the intrinsic structure by the diffusion metric $[23,8,16,25,22]$. In [23], the authors propose a deformation invariant representation of the surface using eigenfunctions and eigenvalues of the Laplace-Beltrami differential operator. They introduced a signature, named GPS, which is then tested with deformed shapes to demonstrate an impressive segmentation. However, the segmentation is not the focus of that paper. The segmentation therefore is simply achieved using Kmeans for grouping the points according to their GPS. The fast mesh segmentation based on random walks is proposed in [16]. The segmentation described in [8] adapts the diffusion maps to aim at a hierarchical segmentation of articulated bodies. The method efficiently segments sequentially articulated bodies in a consistent manner. A most recent work targeting PCMS is introduced in [25]. The persistence-based clustering and heat kernel signature are combined to offer a multi-scale isometry invariant mesh segmentation. While the proposed approaches can address PCMS to some extent, challenges to a robust PCMS method generally come from two sources: the determination of the number of the reasonable segments and the selection of initial seeds for clustering.

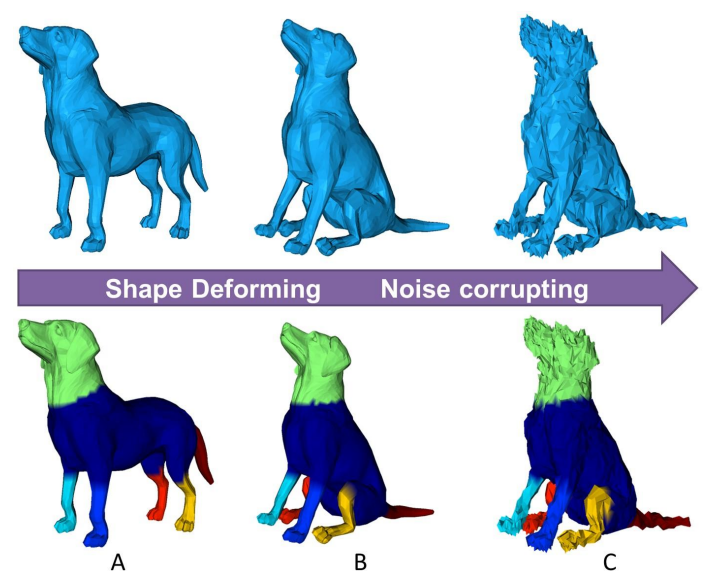

Figure 1. Illustration of PCMS and the performance of HeatMapping.

\subsection{Our solution to PCMS: Heat-Mapping}

In this paper, we introduce a novel approach to address perceptually consistent mesh segmentation by exploring heat kernel featured space to which the geometric features of the mesh are mapped. Along with our pipeline of Heat-Mapping for a robust PCMS, we propose a new pointbased signature, named heat mean signature (HMS), the algorithm for hunting the heat centers, and heat center driven mesh segmentation approach. The HMS follows the similar principle of heat kernel signature (HKS) [26], but interprets the hidden features of mesh structure in a different way by using a physics-based approach using a heat transfer analogy. The heat center is defined as the local maxima of the HMS for all vertices on the surface. We develop a method to locate the heat center based on a greedy algorithm. The heat centers then further drive the mesh segmentation algorithm. Figure 1 illustrates basic ideas about PCMS and the performance of Heat-Mapping. This figure is an overall presentation of the Heat-Mapping algorithm and its performance on deformed models. Figure 1(A) displays a standing dog and the segmentation of the dog is shown underneath. Figure 1(B) displays a sitting dog which is deformed from the standing dog. The segmentation of the sitting dog is illustrated underneath. Figure 1(C) shows a noisy sitting dog model and its segmentation.

The contributions of the paper are five-fold. First, we define perceptually consistent mesh segmentation for lowlevel vision tasks. Second, we develop the heat mean signature (HMS) for characterizing the vertices on a surface in the heat kernel featured space. Third, a new approach for locating heat centers on the surface based on the HMS for the initialization of seeds to further guide mesh segmentation. Fourth, the heat center driven segmentation process for a perceptually consistent segmentation is demonstrated. Fifth, we develop a strategy for the estimation of the opti- 
mal number of the segments based on the observation of the behavior of the Laplacian spectrum.

\section{Methods}

\subsection{Heat Kernel}

The heat kernel quantitatively encodes the heat flow across a manifold $M$ and is uniquely defined for any two vertices $i, j$ on the manifold $[12,3]$. Suppose we apply a unit amount of heat at the node $i$, and allow the heat flow on the manifold across all of the edges. The speed of the diffusion over manifold is determined by the pre-defined weights amongst the edges. The value of heat kernel $H_{t}(i, j)$ is the amount of heat accumulated at $j$ after time $t$. Since it aggregates heat flow through all possible flowing paths between two vertices on the mesh surface, the heat kernel captures much of the structure behind the surface. If a vertex has a number of paths to other vertices, the heat flows faster and aggregates rapidly across the manifold. Consequently, the average temperature is higher when the heat is applied at vertices with a large number of paths to the other vertices. The heat kernel can be approximated by the eigenfunction expansion described below. The 3D model is represented as a graph $G=(V, E, W)$, where $V$ is the set of vertices, $E$ is a set of edges and $W$ is the weight value for each edge. The widely used cotangent weight for the edges is adopted in our work [23]. The graph Laplacian determines the rate of the heat-flow across the weighted graph with time. The solution to the heat equation is found by exponentiating the Laplacian eigensystem with time. The graph Laplacian is defined as follows:

$$
L=D-W
$$

where $D$ is a diagonal degree matrix and its diagonal entries are given by summation of the rows of $W$. The normalized Laplacian is defined as follow:

$$
L=D^{-1 / 2} L D^{-1 / 2}
$$

Then the heat kernel can be defined by

$$
H_{t}(i, j)=\sum_{k=1}^{|V|} \exp \left(-\lambda_{k} t\right) \phi_{k}(i) \phi_{k}(j)
$$

Where $\lambda_{k}$ is the $k_{t h}$ eigenvalue of the Laplacian and $\phi_{k}$ is the $k_{t h}$ eigenfunction. The $H_{t}(i, j)$ is defined as the heat affinity $H_{a f}(i, j)$ between a pair of vertices which is a measure of heat transfer between node $i$ and node $j$ after time $t$.

In addition, we define the heat coordinate to embed the vertices of 3D model in Euclidean space.

$\operatorname{Cor}(i)=\left(e^{-\lambda_{1} t / 2} \phi_{1}(i), e^{-\lambda_{2} t / 2} \phi_{2}(i), \ldots, e^{-\lambda_{k} t / 2} \phi_{k}(i)\right)$

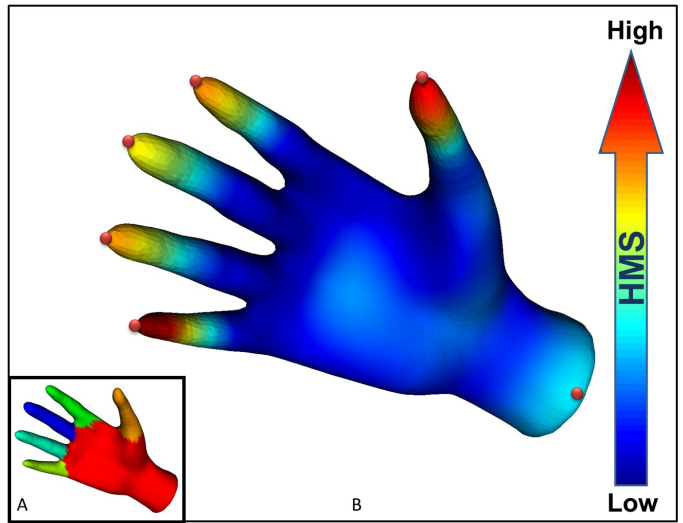

Figure 2. Illustration of Heat Mean Signature (HMS) of a human hand model. Figure (A) displays the six segments of the hand. Figure (B) illustrates the HMS distribution across the mesh surface of a human hand model.

Where $\lambda_{k}$ is the $k_{t h}$ eigenvalue of the Laplacian, $t$ denotes the time of heat dissipating on the manifold and $\phi_{k}$ is the $k_{t h}$ eigenfunction. The heat coordinate is defined on an $n$ dimensional (less than the number of the data points) space.

\subsection{Heat mean signature}

We define a novel one point signature, heat mean signature (HMS), for quantitatively evaluating the temperature distribution resulting from the heat flow process. The HMS has the following formulation:

$$
H M S(i)=\frac{1}{N} \sum_{j, j \neq i} H_{t}(i, j)
$$

where $i$ and $j$ denote the $i t h$ and $j t h$ vertex on the surface, respectively and $N$ is the total number of vertices.

HMS(i) can be physically interpreted as the average temperature on the surface obtained by applying a unit amount of heat on the vertex $i$ and after a certain amount of time of heat dissipation. The HMS has desirable properties which make it powerful in the application of PCMS: 1) It is invariant to near-isometric transformation of the 3D model, 2) It is resistant to numerical and topological noise, 3) It faithfully reflects the temperature distribution during the heat process.

\subsubsection{Heat center and sink}

For each segment, its heat center is defined as the vertex with the highest value of HMS amongst all of its vertices. The rest of the vertices of the segment are defined as the heat sinks for that heat center. The heat center is formulated as:

$$
H_{c}=\underset{V \in V_{c}}{\operatorname{argmax}}\{H M S(V)\}
$$


Where $H_{c}$ denotes the heat center for the segment $C$ and $V_{C}$ denotes the vertex set on $C . H M S(V)$ denotes the heat mean signature for the vertex $V$. Figure 2(B) illustrates the heat mean signature, heat centers, and heat sinks of a human hand model. The HMS is mapped on the surface with the colormap: the higher the value of the HMS, the closer the color is to red. The hand model is segmented into six functional components (see Figure 2(A)). Each finger corresponds to one segment and the palm is partitioned as a separate part. The detected heat centers are highlighted using red balls in the figure. It can be visually observed that the heat centers are associated with the highest HMS within their own segment. For example, there is a heat center located at the tip of each finger in Figure 2(B). The color of the heat sink is closer to the blue end of the colorbar, indicating lower average temperatures.

\subsection{Heat-Mapping Algorithm}

\subsubsection{Estimating number of heat centers}

The estimation of the number of heat centers is equivalent to the estimation of the number of the segments. Here, we describe a simple approach based on the analysis of the behavior of the Laplacian spectrum. A similar observation is mentioned in [17]. Let $0=\lambda_{1}<\lambda_{2} \ldots \lambda_{n}$ be the Laplacian spectrum of the model, where $\lambda_{i}$ is the $i$ th eigenvalue and $\operatorname{Dif}(i, j)=\lambda_{j}-\lambda_{i}$. Then we have the following observation: The number $i$ is likely to coincide with the number of components in a natural segmentation when there is a dramatic increase in $\operatorname{Dif}(i, i+1)$ for the first time. We provide the following six segmentation experiments for verifying the observation. We define the curve plotting the eigenvalue versus the number of segmentations as an Eigencurve. Figure 3 displays six Eigen-curves for the segmentations of different models. The models used in this test are: (A) Woman, (B) Octopus, (C) Glasses, (D) Hand, (E) Ant and $(\mathrm{F})$ Horse. The suggested number of segments is circled on the curve and pointed out by an arrow. As shown in Eigen-curve plots, the suggested numbers of cluster are: 6 , $9,4,6,11$ and 7 , respectively.

\subsubsection{Hunting Heat Centers}

The heat kernel measures the heat affinity between any pair of vertices on the surface. The heat affinity reflects how heat flows across the surface by applying heat source at any vertex. The efficiency of heat diffusion over the surface depends on the vertices of applications of the heat. We are interested in globally locating the optimal vertices (heat centers) of application of heat to obtain an efficient heat flow. We develop an algorithm for globally hunting heat centers as follows.

1. Let $V$ be the set of the $N$ vertices on a meshed surface
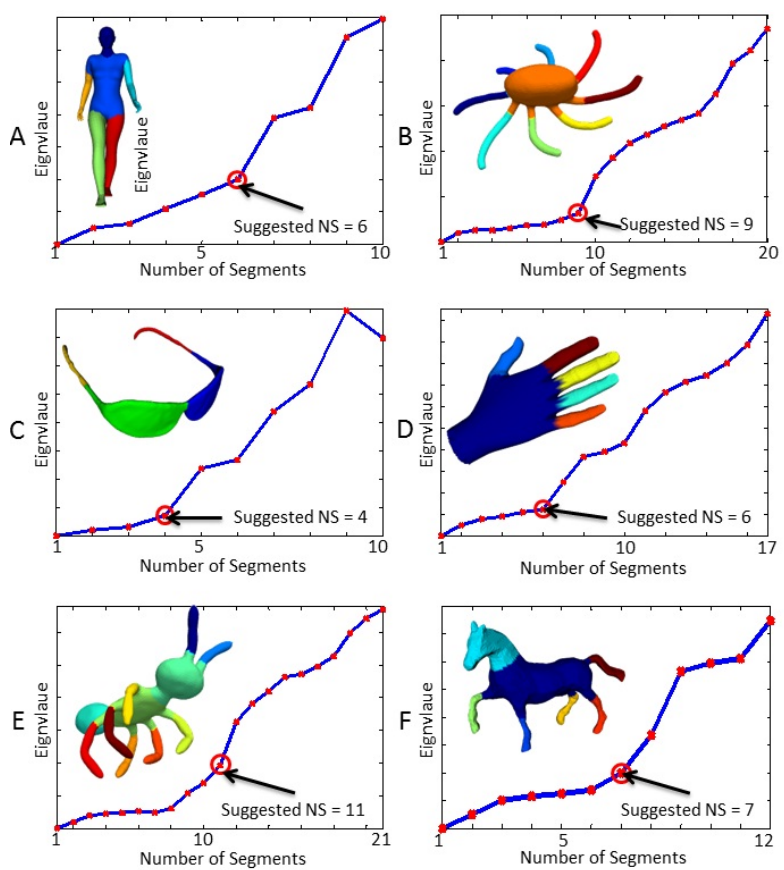

Figure 3. Eigen-curves for estimating perceptually consistent number of segmentations. Eigen-curves plot the relationship between the eigenvalues and the number of segmentations. The first point on the curve with rapid eigenvalue change suggests the number of segments for perceptually consistent mesh segmentation. The point highlighted in red circles on the curves suggest the number of the segments for the models. We can see from the figure that the suggested NS matches the natural number of the segmentation by human perception. Note that, NS denotes number of segments.

$M$. We will find out the number of $C$ heat centers $H_{c}$ on the surface. Let $S_{c}$ be the set of vertices that includes both the heat center $H_{c}$ and its corresponding heat sinks.

2. We define the diameter $D_{c}$ of $S_{c}$ as the largest value of heat affinity for which all of the sinks in $S_{c}$ have heat affinity to $H_{c}$ greater than $D_{c} / 2$.

3. The diameter $D_{M}$ for all of vertices on $M$ is defined in Equation 7:

$$
\begin{aligned}
D_{M} & =\min _{c=1, \ldots, C} D_{c} \\
& =\min _{c=1, \ldots C V_{i} \in S_{c}}\left(H_{a f}\left(V_{i}, H_{c}\right)\right)
\end{aligned}
$$

where $C$ denotes the number of the heat centers on $M, H_{c}$ denotes the heat center, $V_{i}$ denotes $i t h$ vertex denoting the heat sink for its own heat center, $H_{a f}\left(V_{i}, H_{c}\right)$ denotes heat affinity between $V_{i}$ and $H_{c}$

4. Given the number of heat centers, $C$, we maximize the $D_{M}$ to guarantee that the heat affinities among the ver- 
tices are maximized. The heat center hunting problem can be formulated as:

$$
H_{c}=\underset{H_{c}}{\operatorname{argmax}} D_{M}
$$

where $H_{c}$ denotes the heat center, $D_{M}$ is a function of $H_{c}$ (see equation 7), and for each cluster $H_{c}$ is subjected to its defintion in equation 6 .

The numerical implementation is described by the pseudo code in Algorithm 1.

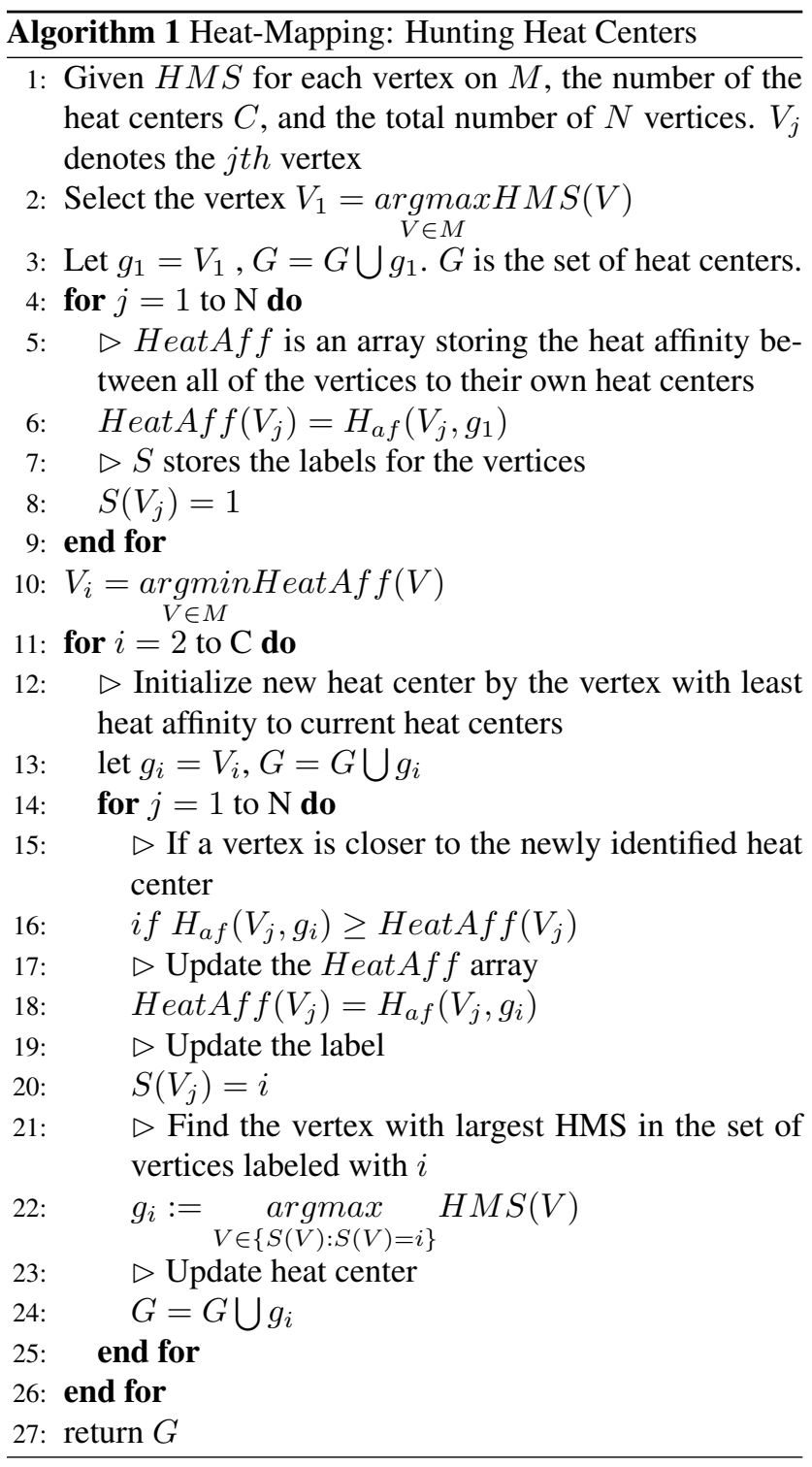

\subsubsection{Heat Center Driven Segmentation}

The mesh segmentation can be treated as the process of grouping similar vertices into geometrically meaningful cliques. There have been a great number of clustering techniques that have been proposed over the past decade [14]. We adopt one of the widely used unsupervised clustering algorithm: Kmeans. Two known limitations of Kmeans arise from its dependence on the number of the clusters and the initialization of the seeds of clusters. We address those two issues by developing a heat-center driven Kmeans (HCKmeans), which gives a stable and efficient clustering procedure. The pseudo code for the algorithm is described in Algorithm 2:

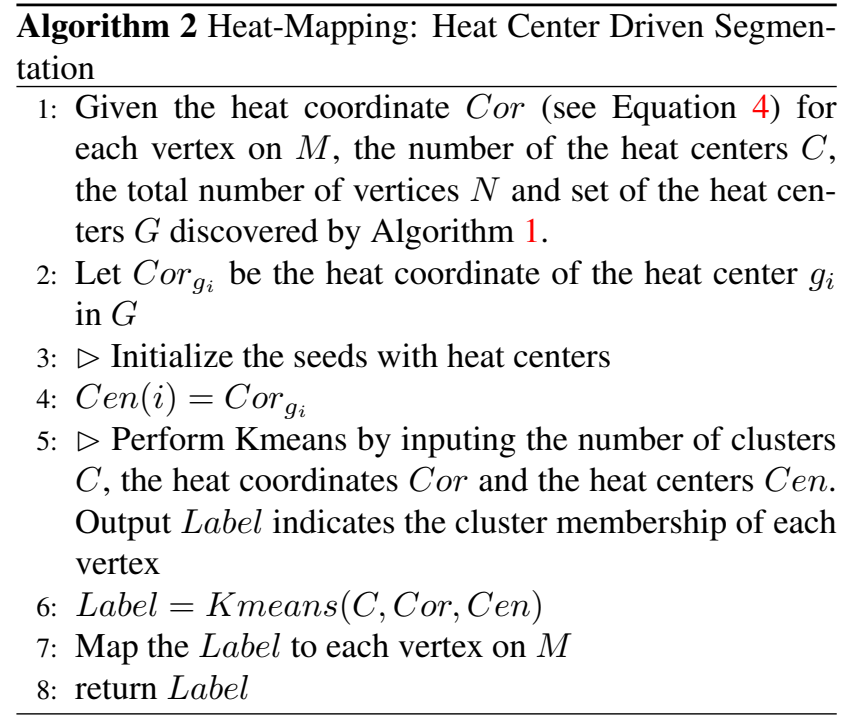

The HC-Kmeans will return a unique label for each vertex so that the vertices can be grouped into different partitions according to the unique label, thus, revealing the segmentation of the model.

\section{Experimental Results}

\subsection{Consistent segmentation of articulated bodies}

In this experiment, we test the performance of HeatMapping on the deformed shape models. We carry out two sets of tests on the centaur model and the armadillo model. The models used in the experiments were chosen from the following databse: ISDB, Princeton Shape Benchmark for 3D Segmentation and TOSCA nonrigid world 3D database $[11,7,5,4,6]$.

Before the test of centaur model, we perform the segmentation of complete human and horse models separately. Then, we segment the centaur model with different poses. This is because the centaur model can be viewed as a mix of human upper body and horse lower body. It is of great 
interest to examine the segmentation consistency at different model settings. The segmentation results are shown in Figure 4). We can see from Figure 4(A) that both the human model and horse model get segmented into seven functional components. Figure 4(B) displays the segmentation results for centaur models at different poses. There are two interesting results based on the comparison of the segmentation shown across Figure 4(A) and (B) and within Figure 4(B). The first comparison indicates a consistent segmentation of the components with common functions. For example, the human head is partitioned out in both Figure 4(A) and (B), and the same goes for the segmentation of the tail of the horse. The second comparison indicates a consistent segmentation of a model with different poses as seen in the six different centaur models, which can be observed from Figure 4(B). In the test of the armadillo model, we focus on the consistent segmentation of incomplete models at different poses. In Figure 5(A), the main features (i.e. torso, head, claws, legs and tail) of the armadillo are correctly extracted and segmenting consistency is also preserved across models at four different poses. We are also interested in the consistency of segmentation between complete and incomplete models. Therefore, we apply Heat-Mapping on the four incomplete armadillo models in this test. The results are shown on Figure 5(B). For example, there is an entirely missing component from the original model, such as missed left arm, right leg or tail. The comparison between the segmentation in Figure 5(A) and (B) demonstrates the consistency. The experiment on various articulated shapes shows that the segmentation results obtained by our method are both perceptually compliant and consistent throughout the test.

\subsection{Consistency over Topological change}

In this test, we will verify the performance of our HeatMapping on the segmentation of the models with topological changes. Figure 6 displays four models among which two models on the left serve as reference model in this experiment. The models on the right have topological noise introduced in different parts are compared with the reference model on the left. For example, the hand on the right has two short links between fingers as indicated in the figure. The dog model on the right has a short link between front two legs. Despite the topology changes, our segmentation results are of high consistency. The experiments on various topologically changing models shows that the segmentation results from our method are perceptually consistent and Heat-Mapping is insensitive to small topological changes.

\subsection{Resistance to noise}

In this experiment, we will demonstrate that HeatMapping is robust to numerical noise. The original model is a human right hand model without noise. Our segmentation result correctly reveals individual fingers and the palm. To investigate the robustness of our method against noise, we further experimented by perturbing the vertices of the original model using various levels of numerical noise. The noise, a 3-dimensional random vector, is generated from a multivariate normal distribution, which can be written using the following notation

$$
\text { Noise } \sim N_{3}(\mu, N S R * \Sigma)
$$

where $\mu=\left[E\left[X_{1}\right], E\left[X_{2}\right], \ldots, E\left[X_{k}\right]\right]$ is the 3-dimensional mean vector (the coordinates of all of the vertices)and $\Sigma=$ $\left[\operatorname{Cov}\left[X_{i}, X_{j}\right]\right]_{i=1,2, \ldots, k ; j=1,2, \ldots, k}$ (the $3 \times 3$ covariance matrix for all of the vertices ), and NSR denotes the ratio between the variance of noise and variance of the original signal (coordinates of the vertices).

We are interested in the consistency of segmentation at different levels of noise. The results of the segmentation of the human hand model at various noise levels are shown on Figure 7. As indicated by the results in the Figure 7, Heat-Mapping performs extremely well at moderate level of noise (e.g. NSR less than 0.01). The segmentation is reasonable even at the exaggerated noise level (e.g. NSR larger than 0.05). As shown in the figure, consistent segmentations are retained even at the noise level with NSR equal to 0.08 . We further increase the noise until Heat-Mapping fails to obtain consistent segmentation. We find that Heat-Mapping fails at noise levels of 0.100 where the geometry has been severely deformed. However, in such conditions, correct segmentation is extremely hard to achieve even based on human perception.

\section{Discussion and Conclusions}

This paper develops a previously undescribed HeatMapping framework that conforms to the perceptually consistent understanding of the mesh models in a robust and intelligent way. The Heat-Mapping exploits the intrinsic structure, and therefore the segmentation result is tolerant to different kinds of noise. In addition, Heat-Mapping segments the mesh model in an assumption free manner as it can estimate the number of the segments of a model. However, we note that Heat-Mapping should not be applied blindly to segmentation. One critical parameter for the Heat-Mapping that has not been discussed in the experiment is the heat dissipation time. The dissipation time is crucial to indicate how locally or globally the geometric structure is exploited. The larger the time, the more globally the structure is exploited. In the future, we will develop a computational scheme to determine the optimal time to describe the structure. However, based on the experiments, the PCMS prefers to small time as the high resolution details are preserved at a small scale. The determination of 


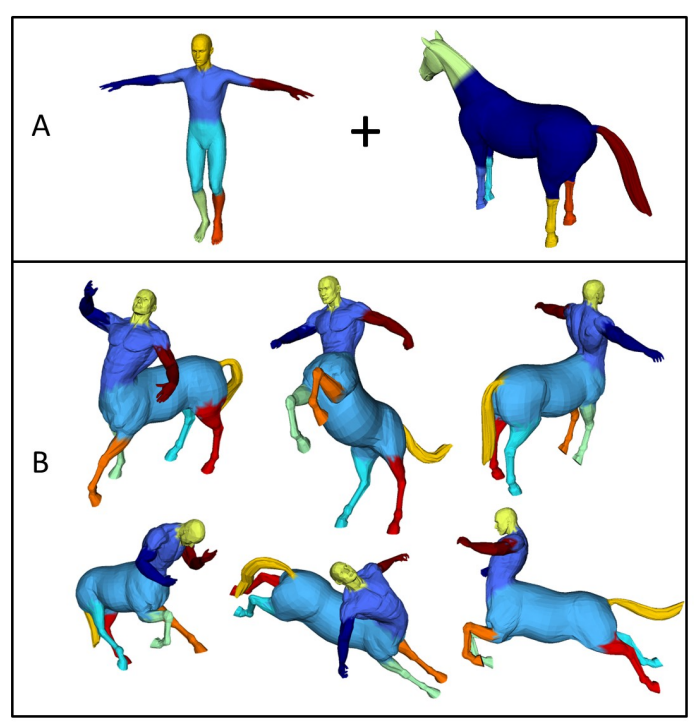

Figure 4. Segmentation results on articulated models. Figure (A) shows the segmentation of human and horse model. Figure (B) displays segmentation results of centaur models with different articulations.

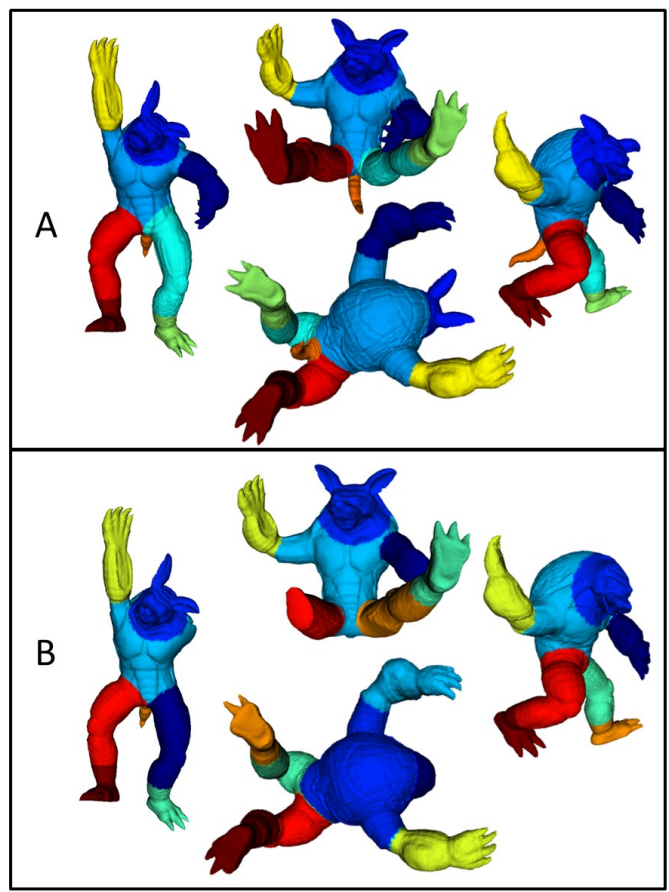

Figure 5. Segmentation results on Armadillo models. Figure (A) illustrates the segmentation result of various articulated Armadillo models. Figure (B) shows the segmentation result on a set of incomplete models which are the deformed version of models in Figure (A). The models in (B) correspond to its original model in (A) but have some missing components such as the left arm, right leg or the tail.

the threshold for the first turning point on the Eigen-curve
A

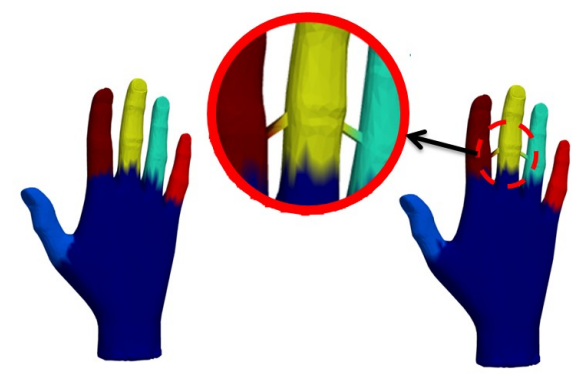

B
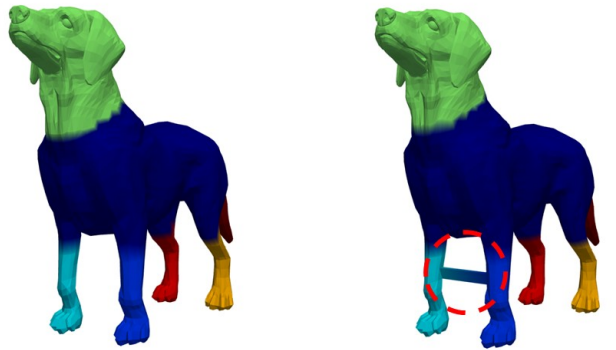

Figure 6. Segmentation result on models with small topological noises induced. The figures on the left show the reference models (a hand and a dog) and their segmentations using Heat-Mapping. Small topological short-circuits are added into the models on the right showing the segmentation of the models with topological short-circuits.

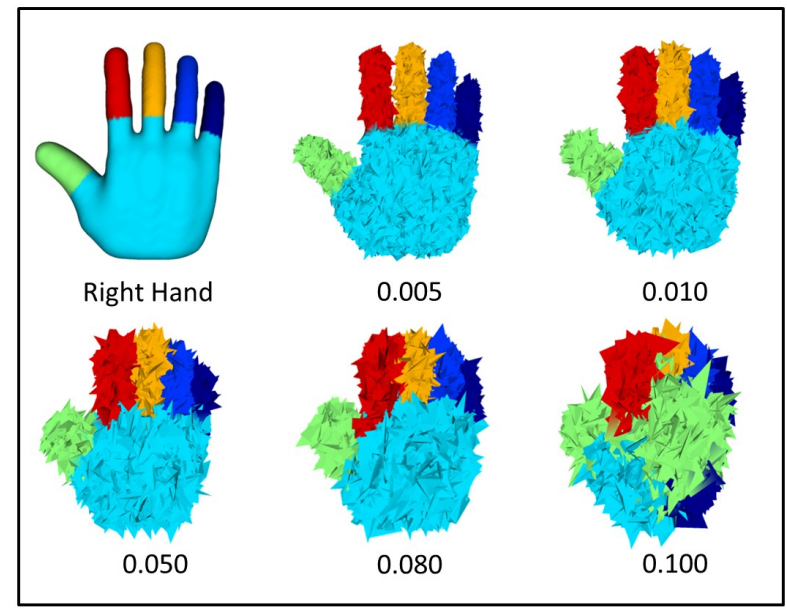

Figure 7. Segmentation results on noise-corrupted models. The top left model is the original human right hand model for reference. The verteices of other models are perturbed with different levels of noise. Our method can work well on exaggerated levels of noise (NSR=0.08). However Heat-Mapping fails at noise levels with NSR equal to 0.100. At these noise levels, even human perception has difficulty in segmenting the model. Note that the value underneath each figure denotes NSR of the noise applied on the model.

will be studied in the future. We will also investigate the segmentation at different turning points on the Eigen-curve for a hierarchical segmentation. In addition, the theoretical proof for the estimation of the number of segments based 
on the Eigen-curve will be further explored.

\section{Acknowledgement}

We would like to thank the Institute for Pure \& Applied Mathematics (IPAM), a National Science Foundation (NSF) Math Institute at the University of California at Los Angeles (UCLA) for the fellowship to support Karthik Ramani. The experimental images are provided by the whole brain database from Harvard university. This work is partly based upon work supported by the National Science Foundation Partnership for Innovation Grant\# 0917959 (3D Hub), NSF IIS Grant\# 0535256, Donald W. Feddersen Chair Professorship support, Purdue University support for Faculty Study in a second discpline, and the School of Mechanical Engineering. Any opinions, findings, and conclusions or recommendations expressed in this material are those of the author(s) and do not necessarily reflect the views of the National Science Foundation.

\section{References}

[1] E. Agathos, I. Pratikakis, S. Perantonis, N. Sapidis, and P. Azariadis. 3d mesh segmentation methodologies for cad applications. Computer-Aid Design and Application, 2007. 2145

[2] M. Attene, S. Katz, M. Mortara, G. Patane, M. Spagnuolo, and A. Tal. Mesh segmentation - a comparative study. Shape Modeling and Applications, 2006. SMI 2006. IEEE International Conference on, pages 7-7, 2006. 2146

[3] M. Belkin, J. Sun, and Y. Wang. Discrete laplace operator on meshed surfaces. SGG 2008 Proceedings of the twentyfourth annual symposium on computational geometry, pages 93-193, 2008. 2147

[4] A. Bronstein, M. Bronstein, and R. Kimmel. Calculus of nonrigid surfaces for geometry and texture manipulation. IEEE Transactions on Visualization and Computer Graphics, 13:902-913, 2007. 2149

[5] A. M. Bronstein, M. M. Bronstein, and R. Kimmel. Efficient computation of isometry-invariant distances between surfaces. SIAM J. Sci. Comput., 28:1812-1836, September 2006. 2145, 2149

[6] A. M. Bronstein, M. M. Bronstein, and R. Kimmel. Numerical geometry of non-rigid shapes. Springer, 2008. 2149

[7] X. Chen, A. Golovinskiy, and T. Funkhouser. A benchmark for 3D mesh segmentation. ACM Transactions on Graphics (Proc. SIGGRAPH), 2009. 2145, 2146, 2149

[8] F. De Goes, S. Goldenstein, and L. Velho. A hierarchical segmentation of articulated bodies. Computer Graphics Forum, 27:1349C1356, 2008. 2145, 2146

[9] H. Edelsbrunner, D. Letscher, and A. Zomorodian. Topological persistence and simplification. Discrete Comput. Geom, 2000. 2146

[10] Y. Fang, Y.-S. Liu, and K. Ramani. Three dimensional shape comparison of flexible proteins using the local-diameter descriptor. BMC Structural Biology, 9(1):29, 2009. 2145
[11] R. Gal, A. Shamir, and D. Cohen-Or. Pose-oblivious shape signature. IEEE Transactions on Visualization and Computer Graphics, 13:261-271, 2007. 2146, 2149

[12] A. Grigoryan. Heat kernels on weighted manifold an application. Contemporary Mathematics, 398:93-193, 2006. 2147

[13] N. Iyer, S. Jayanti, K. Lou, Y. Kalyanaraman, and K. Ramani. Three-dimensional shape searching: state-of-the-art review and future trends. Computer-Aided Design, 37(5):509 - 530, 2005. 2145

[14] A. K. Jain, M. N. Murty, and P. J. Flynn. Data clustering: A review. ACM computing surveys. 2149

[15] S. Katz, G. Leifman, and A. Tal. Mesh segmentation using feature point and core extraction. The Visual Computer, 21:649-658, 2005. 10.1007/s00371-005-0344-9. 2145, 2146

[16] Y.-K. Lai, S.-M. Hu, R. R. Martin, and P. L. Rosin. Fast mesh segmentation using random walks. Proceedings of the 2008 ACM symposium on Solid and physical modeling, pages 183-191, 2008. 2145, 2146

[17] W. Li, W.-K. Ng, and E.-P. Lim. Spectral analysis of text collection for similarity-based clustering. In Data Engineering, 2004. Proceedings. 20th International Conference on, 2004. 2148

[18] Y.-S. Liu, Y. Fang, and K. Ramani. Idss: deformation invariant signatures for molecular shape comparison. BMC Bioinformatics, 10(1):157, 2009. 2145

[19] A. Mangan and R. Whitaker. Partitioning 3d surface meshes using watershed segmentation. Visualization and Computer Graphics, IEEE Transactions on, 5(4):308 -321, 1999. 2146

[20] R. Osada, T. Funkhouser, B. Chazelle, and D. Dokin. Shape distributions. ACM Transactions on Grphics, 33:133-154, 2002. 2145

[21] A. Razdan and M. Bae. A hybrid approach to feature segmentation of triangle meshes. Computer Aided Design, 35(9):783 - 789, 2003. 2146

[22] M. Reuter. Hierarchical shape segmentation and registration via topological features of laplace-beltrami eigenfunctions. International Journal of Computer Vision, 89:287308, 2010. 10.1007/s11263-009-0278-1. 2146

[23] R. M. Rustamov. Laplace-beltrami eigenfunctions for deformation invariant shape representation. Proceedings of the fifth Eurographics symposium on Geometry processing, pages 225-233, 2007. 2145, 2146, 2147

[24] A. Shamir. A survey on mesh segmentation techniques. Computer Graphics Forum, 27(6):1539-1556, 2008. 2145, 2146

[25] P. Skraba, M. Ovsjanikov, F. Chazal, and L. Guibas. Persistence-based segmentation of deformable shapes. Computer Vision and Pattern Recognition Workshops (CVPRW), 2010 IEEE Computer Society Conference on, pages 45 -52, 2010. 2145, 2146

[26] J. Sun, M. Ovsjanikov, and L. Guibas. A concise and provably informative multi-scale signature based on heat diffusion. Computer Graphics Forum, 28:1383-1392, 2009. 2146

[27] E. Zucherberger. Polyhedral surface decomposition with applications. Computers Graphics, 26(5):733-743, 2002. 2146 